BULL. AUSTRAL. MATH. SOC.

\title{
THE $K$-FUNCTIONAL OF CERTAIN PAIRS OF REARRANGEMENT INVARIANT SPACES
}

\author{
JONATHAN ARAZY
}

Let $X, Y$ be rearrangement invariant spaces and let $M=M(Y, X)$ be the space of all multipliers of $Y$ into $X$. It is shown that if $X=Y M$ and some technical conditions are satisfied, then the $K$-functional $K(t, f, X, Y)$ is equivalent to the expression

$$
\left\|f^{*} X_{[0, \psi(t)]}\right\|_{X}+t\left\|f^{*} X[\psi(t), \infty)\right\|_{Y}
$$

where $\psi$ is the inverse of the fundamental function $\varphi_{M}$ of $M$, defined by $\varphi_{M}(u)=\left\|\mathrm{x}_{[0, u)}\right\|_{M}$.

Let $X, Y$ be rearrangement invariant spaces over $[0, \infty$ ) (see [3], $\$ 2 . a$, for basic facts on rearrangement invariant spaces). The $K-$ functional of the pair $(X, Y)$ is defined for every $f \in X+Y$ and $t>0$ by

$$
K(t, f, X, Y)=\inf \left\{\|g\|_{X}+t\|h\|_{Y} ; g \in X, h \in Y, f=g+h\right\}
$$

(see [1] for the application of the $K$-functional in interpolation theory).

In this note we compute, up to equivalence, the $K$-functional of certain pairs $(X, Y)$ of rearrangement invariant spaces, using an auxiliary space of all multipliers from $Y$ into $X$.

We denote by $M=M(Y, X)$ the space of all multipliers from $Y$ into Received 16 November 1982. The author would like to thank Professor Cwikel for bringing references [4], [5], and [6] to the author's attention and for his interest. 
$X$, that is, all measurable functions $f$ so that $f g \in X$ for every $g \in Y$, normed by

$$
\|f\|_{M}=\sup \left\{\|f g\|_{X} ; g \in Y,\|g\|_{Y} \leq 1\right\} .
$$

It is easily verified that $M$ is a Banach lattice (under the pointwise almost everywhere ordering) and that its norm is rearrangement invariant in the sense that if $\tau$ is a measure automorphism of $[0, \infty)$ then $f \in M$ if and only if $f \circ \tau \in M$, and in this case $\|f\|_{M}=\|f \circ \tau\|_{M}$. In what follows we assume, furthermore, that

(a) $M$ is an rearrangement invariant space in the sense of [3], §. a; in particular, $L_{1}(0, \infty) \cap L_{\infty}(0, \infty) \subseteq M$,

(b) the fundamental function $\varphi_{M}(u)=\left\|\mathrm{X}_{[0, u)}\right\|_{M}$ is strictly

$$
\begin{aligned}
& \text { increasing, satisfying } \varphi_{M}(0+)=\lim _{u \rightarrow 0} \varphi_{M}(u)=0 \text { and } \\
& \varphi_{M}\left(\infty_{-}\right)=\lim _{u \rightarrow \infty} \varphi_{M}(u)=\infty,
\end{aligned}
$$

(c) every $f \in X$ has a representation $f=g h$ with $g \in Y$ and $h \in M$, that is, $X=Y M$.

We remark that (b) implies that $M \neq L_{\infty}(0, \infty)$, and thus $X \neq Y$. Also, the fundamental function of every rearrangement invariant space is known to be continuous; thus $\varphi_{M}$ is continuous by (a) (see, for example, [7]).

It follows that the inverse function $\psi=\varphi_{M}^{-1}:[0, \infty) \rightarrow[0, \infty)$ is continuous and strictly increasing, and satisfies

$$
\left\|x_{[0, \psi(t)}\right\|_{M}=t, \quad t>0 .
$$

As usual we denote by $f^{*}$ the decreasing rearrangement of the measurable function $f$ (see [3], \$2.a for basic properties). Our main result is the following theorem.

THEOREM 1. Let $X, Y$ be rearrangement invariant spaces over $[0, \infty)$, and let $M=M(Y, X)$ be the space of all multipliers from $Y$ into $X$. Suppose that properties (a)-(c) are satisfied. Then the $K-$ finctional of the pair $(X, Y)$ is equivalent to the expression 


$$
F(t, f, X, Y)=\left\|f^{*} \chi_{[0, \psi(t)]}\right\|_{X}+t\left\|f^{*} \chi_{[\psi(t), \infty)}\right\|_{Y} \cdot
$$

Precisely, there exists a constant $A>0$ depending only on $X$ and $Y$, so that, for every $f \in X+Y$ and every $t>0$,

$$
A^{-1} \cdot F(t, f, X, Y) \leq K(t, f, X, Y) \leq F(t, f, X, Y) .
$$

Before we proceed with the proof, we consider the most important case where $X=L_{p}(0, \infty)$ and $y=L_{q}(0, \infty)$. If $1 \leq p<q \leq \infty$ then $M=M\left(L_{q}, L_{p}\right)=L_{p}$, where $r^{-1}+q^{-1}=p^{-1}$. Properties (a)-(c) hold, and $\varphi_{M}(u)=u^{1 / r}$. Thus $\psi(t)=t^{r}$, and Theorem 1 says that

$$
K\left(t, f, L_{p}, L_{q}\right) \approx\left(\int_{0}^{t^{r}} f^{*}(s)^{p} d s\right)^{1 / p}+t\left(\int_{t^{r}}^{\infty} f^{*}(s)^{q} d s\right)^{1 / q},
$$

which is the well known result of Holmstedt [2, Theorem 4.1].

If $q=\infty$, then one gets easily (see [1])

$$
K\left(t, f, L_{p}, L_{\infty}\right) \approx\left(\int_{0}^{t^{p}} f^{*}(s)^{p} d s\right)^{1 / p} .
$$

If $1 \leq q<p \leq \infty$ then $M=M\left(L_{q}, L_{p}\right)=\{0\}$, so (a)-(c) fail. But in this case we can use the general formula

$$
K(t, f, X, Y)=t K\left(t^{-1}, f, Y, K\right)
$$

to compute the $K$-functional of $\left(L_{p}, L_{q}\right)$ in terms of that of $\left(L_{q}, L_{p}\right)$.

Next we shall need the following facts.

PROPOSITION 2. Let $f_{1}, f_{2} \in L_{1}(0, \infty)+L_{\infty}(0, \infty)$, and let $0<t_{1}, t_{2}$. Then

(i) $\left(f_{1}+f_{2}\right) *\left(t_{1}+t_{2}\right) \leq f^{*}\left(t_{1}\right)+f^{*}\left(t_{2}\right)$,

(ii) $\left(f_{1} \cdot f_{2}\right) *\left(t_{1}+t_{2}\right) \leq f^{*}\left(t_{1}\right) \cdot f^{*}\left(t_{2}\right)$.

Indeed, this follows from the obvious formulas 


$$
\text { (i') } \begin{aligned}
\left\{s ; \mid f_{1}(s)+\right. & \left.f_{2}(s) \mid>f_{1}^{*}\left(t_{1}\right)+f_{2}^{*}\left(t_{2}\right)\right\} \\
& \leq\left\{s ;\left|f_{1}(s)\right|>f_{1}^{*}\left(t_{1}\right)\right\} \cup\left\{s ;\left|f_{2}(s)\right|>f_{2}^{*}\left(t_{2}\right)\right\}
\end{aligned}
$$

and

$$
\text { (ii') } \begin{aligned}
&\left\{s ;\left|f_{1}(s) f_{2}(s)\right|>f_{1}^{*}\left(t_{1}\right) \cdot f_{2}^{*}\left(t_{2}\right)\right\} \\
& \subseteq\left\{s ;\left|f_{1}(s)\right|>f_{1}^{*}\left(t_{1}\right)\right\} \cup\left\{s ;\left|f_{2}(s)\right|>f_{2}^{*}\left(t_{2}\right)\right\}
\end{aligned}
$$

and the definition of the decreasing rearrangement in terms of the distribution function; see [3], §2.a.

PROPOSITION 3. Let $X, Y$ and $M$ be as above, and suppose that $X=Y M$. Then there exists a constant $1 \leq B<\infty$ so that for every nonnegative, non-increasing function $f \in X$ there exist non-negative, nonincreasing functions $g \in Y$ and $h \in M$ so that $f \leq g h$ and $\|g\|_{Y} \cdot\|h\|_{M} \leq B\|f\|_{X}$.

We shall prove Proposition 3 later on.

Finally, recall that for every $0<s<\infty$ the dilation operator $D_{s}$ is defined by

$$
\left(D_{s} f\right)(t)=f(t / s), 0 \leq t<\infty
$$

and is bounded in any rearrangement invariant space $X$. We denote by $\left\|D_{s}\right\|_{X}$ the norm of $D_{s}$ as an operator on $X$.

Proof of Theorem 1. Fix $0<t$ and $f \in X+Y$. Let

$$
K(t, f)=K(t, f, X, Y) \text { and } F(t, f)=F(t, f, X, Y) \text {. }
$$

Clearly $K(t, f)=K\left(t, f^{*}\right)$ and $F(t, f)=F\left(t, f^{*}\right)$ so there is no loss of generality in assuming that $f=f^{*}$; that is, $f \geq 0$ and $f$ is nonincreasing. For every measurable set $E$ we have $f=f \chi_{E}+F_{\chi_{E}}$ and thus

$$
K(t, f) \leq\left\|f \chi_{E}\right\|_{X}+t\left\|f \chi_{\sim E}\right\|_{Y}
$$

Using this with $E=[0, \psi(t))$ we get $K(t, f) \leq F(t, f)$ (where the right hand side may, a priori, be infinite). For the converse inequality, suppose that $f=g+h$ is an arbitrary decomposition with $g \in X$ and $h \in Y$. Then, using Proposition $2(i)$, we get, for every $0<\alpha<1$, 


$$
f(s)=f^{*}(s) \leq g^{*}((1-\alpha) s)+h^{*}(\alpha s)=D_{1 /(1-\alpha)}\left(g^{*}\right)(s)+D_{1 / \alpha}\left(h^{*}\right)(s) .
$$

So, with $\dot{u}=\psi(t)$, we have

$$
\begin{aligned}
\left\|f \chi_{[0, u)}\right\|_{X} & \leq\left\|D_{1 /(1-\alpha)}\left(g^{*}\right) \cdot \chi_{[0, u)}\right\|_{X}+\left\|D_{1 / \alpha}\left(h^{*}\right) \cdot \chi_{[0, u)}\right\|_{X} \\
& \leq\left\|D_{1 /(1-\alpha)}\right\|_{X}\|g\|_{X}+\left\|D_{1 / \alpha}\right\|_{Y}\|h\|_{Y}\left\|x_{[0, u)}\right\|_{M} \\
& \leq \max \left\{\left\|D_{1 /(1-\alpha)}\right\|_{X},\left\|D_{1 / \alpha}\right\|_{Y}\right\} \cdot\left(\|g\|_{X}+t\|h\|_{Y}\right\} .
\end{aligned}
$$

Also

$$
\begin{aligned}
t\left\|f X_{[u, \infty)}\right\|_{Y} & \leq t\left(\left\|D_{1 /(1-\alpha)}\left(g^{*}\right) X_{[u, \infty)}\right\|_{Y}+\left\|D_{1 / \alpha}\left(h^{*}\right) X_{[u, \infty)}\right\|_{Y}\right) \\
& \leq\left\|X_{[0, u)}\right\|_{M}\left\|D_{1 /(1-\alpha)}\left(g^{*}\right) X_{[u, \infty)}\right\|_{Y}+\left\|D_{1 / \alpha}\right\|_{Y} t\|h\|_{Y} .
\end{aligned}
$$

Notice that $g_{0}=D_{1 /(1-\alpha)}\left(g^{*}\right) \in X$ is non-negative and non-increasing. So by Proposition 3 there exist non-negative, non-increasing functions $g_{1} \in Y$ and $g_{2} \in M$ with $g_{0} \leq g_{1} g_{2}$ and $\left\|g_{1}\right\|_{Y}\left\|g_{2}\right\|_{M} \leq B\left\|g_{0}\right\|_{X}$.

It follows that

$$
\begin{aligned}
\left\|\mathrm{x}_{[0, u)}\right\|_{M}\left\|g_{0} \mathrm{x}_{[u, \infty)}\right\|_{Y} & \leq\left\|\mathrm{x}_{[0, u)}\right\|_{M}\left\|g_{1} g_{2} \mathrm{x}_{[u, \infty)}\right\|_{Y} \\
& \leq\left\|\mathrm{x}_{[0, u)}\right\|_{M} g_{2}(u)\left\|g_{1} \mathrm{x}_{[u, \infty)}\right\|_{Y} \\
& \leq\left\|g_{2} \mathrm{x}_{[0, u)}\right\|_{M}\left\|g_{1}\right\|_{Y} \\
& \leq\left\|g_{2}\right\|_{M}\left\|g_{1}\right\|_{Y} \leq B\left\|_{0}\right\|_{X} \leq B\left\|D_{I /(1-\alpha)}\right\|_{X}\|g\|_{X} .
\end{aligned}
$$

Combining (2) and (3) we get

$$
t\left\|f X_{[u, \infty)}\right\|_{Y} \leq B \cdot \max \left\{\left\|D_{1 /(1-\alpha)}\right\|_{X},\left\|D_{1 / \alpha}\right\|_{Y}\right\} \cdot\left(\|g\|_{X}+t\|h\|_{Y}\right),
$$

and so, by (1),

$$
\begin{aligned}
F(t, f) & =\left\|f \chi_{[0, u)}\right\|_{X}+t\left\|f \chi_{[u, \infty)}\right\|_{Y} \\
& \leq 2 B \cdot \max \left\{\left\|D_{1 /(1-\alpha)}\right\|_{X},\left\|D_{1 / \alpha}\right\|_{Y}\right\} \cdot\left(\|g\|_{X}+t\|h\|_{Y}\right) .
\end{aligned}
$$

Taking infimum over all representations $f=g+h$ with $g \in X$ and $h \in Y$ we get

$$
F(t, f) \leq A K(t, f)
$$

where

$$
A=2 B \cdot \inf _{0<\alpha<1}\left(\max \left\{\left\|D_{1 /(1-\alpha)}\right\|_{X},\left\|D_{1 / \alpha}\right\|_{Y}\right\}\right)
$$


This completes the proof of Theorem 1 .

Proof of Proposition 3 . We show first that there exists a constant $B_{1}>0$ so that every $f \in X$ admits a representation $f=g h$ with $g \in Y$ and $h \in M$ and

$$
\|g\|_{Y}\|h\|_{M} \leq B_{I}\|f\|_{X}
$$

Indeed if there is no such constant then there exist a sequence $\left\{E_{n}\right\}_{n=1}^{\infty}$ of disjoint measurable sets of positive measure, and a sequence $\left\{f_{n}\right\}_{n=1}^{\infty}$ of functions in $X$ satisfying $f_{n}=f_{n} x_{E_{n}},\left\|f_{n}\right\|_{X}=1$, and so that if $f_{n}=g h$ with $g=g X_{E_{n}} \in Y$ and $h=h \chi_{E_{n}} \in M$ then $\|g\|_{Y}\|h\|_{M} \geq n^{3}$. Let $f=\sum_{n=1}^{\infty} f_{n} / n^{2}$. Then $f \in X$ and so $f=g h$ with $g \in Y$ and $h \in M$. Since $f_{n} / n^{2}=g X_{E_{n}} \cdot h X_{E_{n}}$, we get, for every $n$,

$$
n^{3} \leq n^{2}\left\|g \chi_{E_{n}}\right\|_{Y}\left\|h \chi_{E_{n}}\right\|_{M} \leq n^{2}\|g\|_{Y}\|h\|_{M}
$$

which is obviously a contradiction.

Next suppose that $f \in X$ is non-negative and on-increasing. By the first step of the proof, $f=g h$, with $g \in Y, h \in M$ and $\|g\|_{Y}\|h\|_{M} \leq B_{1}\|f\|_{X}$. By Proposition 2 (ii),

$$
f(s) \leq\left(D_{\frac{1}{2}} g^{*}\right)(s) \cdot D_{\frac{1}{2}}\left(h^{*}\right)(s) .
$$

Clearly $g_{1}=D_{\frac{1}{2}}\left(g^{*}\right) \in Y$ and $h_{1}=D_{\frac{1}{2}}\left(h^{*}\right) \in M$ are both non-negative and non-increasing, and

$$
\begin{aligned}
\left\|g_{1}\right\|_{Y}\left\|h_{1}\right\|_{M} & \leq\left(\left\|D_{\frac{1}{2}}\right\|_{Y}\|g\|_{Y}\right)\left(\left\|D_{\frac{1}{2}}\right\|_{M}\|h\|_{M}\right) \\
& \leq\left(B_{1}\left\|D_{\frac{1}{2}}\right\|_{Y} \cdot\left\|D_{\frac{1}{2}}\right\|_{M}\right)\|f\|_{X} .
\end{aligned}
$$

This completes the proof of Proposition 3, with $B=B_{1}\left\|D_{\frac{1}{2}}\right\|_{Y} \cdot\left\|D_{\frac{1}{2}}\right\|_{M}$. (Notice that since $\|g h\|_{X} \leq\|g\|_{Y}\|h\|_{M}$ for every $g \in Y$ and $h \in M$, we have $B \geq 1$. )

Let us apply Theorem 1 to an important special case, generalizing 
Holmstedt's result [2]. Let $X$ be a minimal rearrangement invariant space over $[0, \infty)$ so that $\varphi_{X}(u)=\left\|X_{[0, u)}\right\|_{X}$ is strictly increasing and satisfies $\lim _{u \rightarrow 0} \varphi_{X}(u)=0, \lim _{u \rightarrow \infty} \varphi_{X}(u)=\infty$. For every $0<\alpha \leq 1$ let

$$
x^{\alpha}=\left\{f ;|f|^{1 / \alpha} \in X\right\}
$$

normed by

$$
\|f\|_{X}^{\alpha}=\left\||f|^{I / \alpha}\right\|_{X}^{\alpha}
$$

It is well known that $x^{\alpha}$ is a rearrangement invariant space (identified with the p-convexification of $X$, where $\alpha=1 / p)$. Moreover, if $X$ is reflexive, then $x^{\alpha}=\left(L_{\infty}(0, \infty), X\right)_{\alpha}$. We define $x^{0}=L_{\infty}(0, \infty)$.

PROPOSITION 4. Let $X$ be as above and let $0 \leq \beta<\alpha \leq 1$. Then

(i) $M=M\left(x^{\beta}, x^{\alpha}\right)=x^{\alpha-\beta}$, and properties (a)-(c) hold for the pair $\left(x^{\alpha}, X^{\beta}\right)$,

(ii) $K\left(t, f, x^{\alpha}, x^{\beta}\right)$ is equivalent to the expression

$$
\begin{aligned}
F\left(t, f, X^{\alpha}, X^{\beta}\right) & =\left\|f^{*} \chi[0, \psi(t))\right\|_{X}^{\alpha}+t \| f^{*} \chi[\psi(t), \infty){ }_{X}^{\beta} \\
& =\left\|\left(f^{*}\right)^{1 / \alpha} x[0, \psi(t)]\right\|_{X}^{\alpha}+t\left\|\left(f^{*}\right)^{1 / \beta} x[\psi(t), \infty)\right\|_{X}^{\beta} \\
\text { where } \psi(t) & =\varphi_{X}^{-1}\left(t^{1 /(\alpha-\beta)}\right) .
\end{aligned}
$$

Proof. We prove only the fact that $M=x^{\alpha-\beta}$ in the case $\beta>0$; the rest follows easily from our assumptions on $X$ and Theorem 1 (notice that if $\beta=0$ then $\left.M=x^{\alpha}\right)$. Let $f \in x^{\alpha-\beta}$ and $g \in x^{\beta}$. Then $f_{1}=|f|^{1 /(\alpha-\beta)}$ and $g_{1}=|g|^{1 / \beta}$ belong to $X$, and thus (see [3], p. 43)

$$
|f g|^{1 / \alpha}=f_{1}^{1-(\beta / \alpha)} \cdot g_{1}^{\beta / \alpha} \in X
$$

that is, $f g \in \chi^{\alpha}$, and 


$$
\begin{aligned}
\|f g\|_{X}^{\alpha} & =\left\|f_{I}^{1-(\beta / \alpha)} \cdot g_{I}^{\beta / \alpha}\right\|_{X}^{\alpha} \\
& \leq\left\|f_{1}\right\|_{X}^{\alpha-\beta} \cdot\left\|g_{1}\right\|_{X}^{\beta}=\|f\|_{X}^{\alpha-\beta} \cdot\|g\|_{X}^{\beta} \cdot
\end{aligned}
$$

This shows that $f \in M$ and $\|f\|_{M} \leq\|f\|_{X^{\alpha-\beta}}$. For the converse inclusion, let $f \in L_{1}(0, \infty) \cap L_{\infty}(0, \infty)$ be a non-negative function. Let $s>0$ be defined by $(1+s) / \alpha=s / \beta$. Then

$$
\left\|f^{l+s}\right\|_{X^{\alpha}} \leq\|f\|_{M}\left\|f^{s}\right\|_{X^{\beta}}=\|f\|_{M}\left\|f^{s / \beta}\right\|_{X}^{\beta}
$$

Thus

$$
\left\|f^{(1+s) / \alpha}\right\|_{X}^{\alpha} \leq\|f\|_{M}\left\|f^{(1+s) / \alpha}\right\|_{X}^{\beta}
$$

so, by the definition of $s$,

$$
\left\|f^{\alpha-\beta}\right\|_{X}^{\alpha-\beta}=\|f\|_{X}^{\alpha-\beta} \leq\|f\|_{M}
$$

By minimality of $X$ this implies that $M \subseteq X^{\alpha-\beta}$, and thus $M=X^{\alpha-\beta}$ (with equality of norms).

REMARK 1. Theorem I holds, with obvious modifications, for rearrangement invariant spaces over $N=\{0,1,2, \ldots\}$, that is, for symmetric sequence spaces.

REMARK 2. After completing the first draft of the present paper, we learned from M. Cwikel that some (slightly weaker) versions of our Theorem 1 have already been established by different methods in [6], [4], and [5]. Our proof avoids the use of the auxiliary spaces of the form $M(X)$ and $\Lambda(X)$, as well as the hypothesis that $\varphi_{M}(u) / u^{r}$ is increasing for some $r>0$.

We conclude the paper with the following problem.

PROBLEM. Find an explicit formula for the $K$-functional of a general pair $(X, Y)$ of rearrangement invariant spaces on $\Omega$, where $\Omega$ is either $N$ or $[0,1]$ or $[0, \infty)$. In particular, does there exist for every $t>0$ a measurable set $A=A_{t} \subseteq \Omega$, so that 


$$
\begin{aligned}
& \text { Rearrangement invariant spaces } \\
& K(t, f, X, Y) \approx\left\|f^{*} X_{A}\right\|_{X}+t\left\|f^{*} X_{\Omega \backslash A}\right\|_{Y}
\end{aligned}
$$

for every $f \in X+Y$ ?

We suggest the use of the function $\left\|D_{u}\right\|_{X} /\left\|D_{u}\right\|_{Y}$ instead of our $\varphi_{M}(u)$, or Milman's $s(u)=\varphi_{X}(u) / \varphi_{Y}(u)$.

\section{References}

[1] Jöran Bergh, Jörgen Löfström, Interpolation spaces. An introduction (Die Grundlehren der mathematischen Wissenschaften, 223. Springer-Verlag, Berlin, Heidelberg, New York, 1976).

[2] Tord Holmstedt, "Interpolation of quasi-normed spaces", Math. Scand. 26 (1970), 177-199.

[3] Joram Lindenstrauss, Lior Tzafriri, Classical Banach spaces II . Function spaces (Ergebnisse der Mathematik und ihrer Grenzgebiete, 97. Springer-Verlag, Berlin, Heidelberg, New York, 1979).

[4] Mario Milman, "Interpolation of operators of mixed weak-strong type between rearrangement invariant spaces", Indiana Univ. Math. J. 28 (1979), 985-992.

[5] Mario Milman, "The computation of the $K$-functional for couples of rearrangement invariant spaces", submitted.

[6] Alberto Torchinsky, "The $K$-functional for rearrangement invariant spaces", Studia Math. 64 (1979), 175-190.

[7] M. Zippin, "Interpolation of operators of weak type between rearrangement invariant function spaces", J. Funct. Anal. 7 (1971), 267-284.

Department of Mathematics, University of Haifa,

Haifa, Israel. 